\title{
Significance of EGFR/HER2 Expression and PIK3CA Mutations in Giant Cell Tumour of Bone Development
}

\author{
Raja Amri (iD, ${ }^{1,2}$ Slim Charfi, ${ }^{3}$ Mohamed Jemaà $\left(\mathbb{D},{ }^{4}\right.$ Nabil Miled, ${ }^{5}$ Fathia Slimi $\left(\mathbb{D},{ }^{2}\right.$ \\ Mohamed Ali Rebai, ${ }^{2}$ Mayssa Abdelwahed, ${ }^{6}$ Hassib Keskes, ${ }^{2}$ and Sami Aifa ${ }^{1}$ \\ ${ }^{1}$ Laboratory of Molecular and Cellular Screening Processes, Centre of Biotechnology of Sfax, Sidi Mansour Road Km 6, BP 1177, \\ 3018 Sfax, Tunisia \\ ${ }^{2}$ Experimental Surgery of the Musculoskeletal System, Faculty of Medicine of Sfax, Tunisia \\ ${ }^{3}$ Department of Pathology, Habib Bourguiba Hospital, Road El Ain, 3029 Sfax, Tunisia \\ ${ }^{4}$ Department of Laboratory Medicine, Translational Cancer Research, Lund University, Lund 22381, Sweden \\ ${ }^{5}$ Faculty of Sciences, Department of Biological Sciences, University of Jeddah, Jeddah, KSA, Tunisia \\ ${ }^{6}$ Medical Genetics Department of Hedi Chaker Hospital, Route El Ain, Sfax 3089, Tunisia \\ Correspondence should be addressed to Raja Amri; raja.amri89@hotmail.fr
}

Received 24 January 2020; Revised 8 June 2020; Accepted 16 July 2020; Published 5 August 2020

Academic Editor: Despina Deligianni

Copyright (c) 2020 Raja Amri et al. This is an open access article distributed under the Creative Commons Attribution License, which permits unrestricted use, distribution, and reproduction in any medium, provided the original work is properly cited.

\begin{abstract}
Giant Cell Tumour of Bone (GCTB) is a rare bone tumour. Locally aggressive and recurrent, it might evolve into pulmonary metastases. Our present work is aimed at investigating the involvement of the epidermal growth factor receptor (ErbB) family and its downstream effectors in the development and recurrence of GCTB. For this purpose, we used a cohort of 32 GCTB patients and we evaluated the clinicohistological features and the expression of RANKL, EGFR, and HER2. The mutation status of KRAS, PI3KCA, and PTEN gene as potential oncogene involved in GCTB was also evaluated. We found a significant correlation between advanced histological stages, overexpression of EGFR/HER2, and tumour recurrence. Moreover, two mutations were found in the PIK3CA gene: a missense mutation, $1634 \mathrm{~A}>\mathrm{C}$, detected for the first time in GCTB patients, without influencing the stability of the protein, and a frameshift mutation, c.1658_1659delGTinsC, causing the loss of the protein kinase domain. Altogether, these results suggest that overexpression of HER2/EGFR, Campanacci, and histological stages could be used as a novel prognostic marker for GCTB recurrence.
\end{abstract}

\section{Introduction}

Giant Cell Tumour of Bone (GCTB) is an intermediate locally aggressive primary bone tumour. Due to its local recurrence and rare pulmonary metastases, GCTB is considered to have a low malignant potential [1,2]. Aggressiveness characteristics include a high mitotic rate, necrosis, and recurrence after resection [3]. Local recurrence after surgery is estimated to occur in $15-50 \%$ of patients, with a higher prevalence after curettage than after en bloc resection [4]. Recurrence of GCTB is not fatal in most cases but can lead to disability and to a poor quality of life as a result of repeated and radical operations, loss of bone stock, and secondary arthritis of the joints [5]. In United States,
GCTB accounts for approximately 3 to $5 \%$ of all primary bone tumours and 15 to $20 \%$ of all benign bone tumours $[6,7]$. A slightly higher incidence was suggested in a population-based series from the Swedish Cancer Registry; of the 4625 bone tumours diagnosed over a 53-year period, 505 (11\%) were GCTB [8]. Asian populations have a significantly higher incidence than Western populations. In China, GCTB represents approximately $20 \%$ of all primary bone tumours $[9,10]$.

GCTB usually occurs in patients between 20 and 40 years old with a slightly higher rate in women than men (1.5 to 1 ratio) [11]. GCTB is formed by multinuclear osteoclast-like cells. These cells are originated essentially from macrophages and monocytes stimulated by various cytokines [12]. GCTB 
is also formed by stromal cells which have been identified as the neoplastic cell population and evolved from mesenchymal stem cells $[13,14]$. Identifying risk factors influencing recurrence in GCTB is still nonwell developed. Previous studies reported that recurrence is influenced by clinical features, such as gender, age, location, tumour size, and surgery method [15]. Moreover, certain factors were proposed as predictors of recurrence and aggressiveness markers in GCTB. Indeed, the overexpression of interleukin-17A (IL-17A) and $\beta$-catenin is closely associated with GCTB progression and recurrence [16]. In addition, it has been reported that Ezrin, VEGF, and CD44 play a role in invasion, metastasis, and recurrence in GCTB [17]. A recent study has indicated that postsurgery fluids are an adjuvant in the mechanism of tumour recurrence, increasing stem cell growth and AKT/mTOR activity [18].

A strong correlation exists between solid cancers and the overexpression of the ErbB receptors $[19,20]$. However, this correlation was not studied in GCTB. The ErbB family members, especially EGFR and HER2, activate the proliferative signalling through the MAPkinase and the PI3kinase pathways [21]. ErbB downstream signalling such as PI3K/AKT and mitogen-activated protein kinase (MAPK) pathways was involved in bone loss. In fact, during osteolysis, the activation of nuclear factor $-\kappa \mathrm{B}(\mathrm{NF}-\kappa \mathrm{B}), \mathrm{MAPK}$, and PI3K/AKT signalling pathways is triggered by RANKL, inflammatory factors, and oxidative stress [22]. It has been demonstrated that PI3K and its downstream target AKT (serine/threonine kinase) play a significant role in the differentiation of progenitor cells into mature osteoblasts [23]. Moreover, the $\mathrm{PI} 3 \mathrm{~K} / \mathrm{AKT}$ and MAPK pathways ensure specific roles in osteoblast differentiation and in RANKL-induced osteoclastogenesis [24-26].

$\mathrm{PI} 3 \mathrm{~K} / \mathrm{AKT}$ and RAS/RAF/MEK/ERK pathways regulate certain cellular functions, including cell proliferation, differentiation, survival, and apoptosis [27]. PTEN (phosphatase and TENsin homolog), a tumour suppressor gene, exerts a negative control of the phosphoinositide 3-kinase signalling pathway and cell proliferation/survival by dephosphorylating the phosphatidylinositol 3,4,5-triphosphate [28-30]. Deregulation of these pathways leads to the occurrence and progression of certain cancers mainly by somatic mutations [31, 32]. The most common PIK3CA mutations had been reported in exons 9 and 20, representing different hotspot mutations [33]. There are 3 hotspot mutations in PIK3CA gene: E542K, E545K at exon 9 (helix domain), and H1047R at exon 20 (kinase domain) [34]. The 3 hotspots represent almost $80 \%$ of PIK3CA mutations and lead to constitutive PI3K activity by different mechanisms [35]. KRAS is an oncogene playing a significant role in downstream signalling of the EGFR pathway. KRAS mutations are observed in many human malignancies, such as non-small-cell lung cancer, colorectal cancer, and pancreatic ductal adenocarcinoma [36-39]. KRAS mutations commonly arise in codon 12 and 13 of exon 2 [40]. Considering all this background, a possible overexpression of RANK/RANKL and members of the ErbB receptor family in GCTB, in addition to the involvement of PIK3CA, PTEN, and KRAS mutations, is suggested to influence GCTB pathogenesis.
For this purpose, we collected a cohort of GCTB patients within the region of Sfax, South Tunisia, and we performed several investigations, including clinical and histological characterisation, and mutational analysis. The main goal was to propose a novel prognostic marker for GCTB recurrence.

\section{Materials and Methods}

2.1. Clinical Cases and Samples. 32 patients with GCTB (11 men, 21 women) were recruited in the Orthopaedics and Traumatology Department, Habib Bourguiba University Hospital, Sfax. From 2015 to 2017, complete clinical and pathological data were generated for each patient. Biopsies were collected after surgery and prepared for further analysis. Informed consent was obtained for all individual participants included in the study.

2.2. Tumour Morphology. Formalin-Fixed ParaffinEmbedded (FFPE) tissue sections $(4-5 \mathrm{~mm})$ were deparaffinised, hydrated, and stained with hematoxylin and eosin (H\&E). Baseline and on-study tissue samples from each patient were evaluated for changes in overall tissue composition and architecture by light microscopy. Then, the collected tumours were graded according to the "Campanacci" and “Jaffe-Lichtenstein" grading.

2.3. Campanacci Grading. GCTBs were classified by Campanacci et al. based on radiographic appearance: stage I: latent, stage II: active, and stage III: aggressive [41].

2.4. Jaffe-Lichtenstein Grading. The histological grading system of Jaffe et al. can be summarized as follows: For grade 1 , tumours are the least aggressive and show a moderate loss, with vascular stroma composed of spindle and ovoid cells, with few or no mitotic figures and numerous giant cells. For grade 2, tumours manifest, cytologically, a very compact cellular stroma showing definite evidence of atypia, tend strongly toward recurrence, and in some cases eventually undergo malignant transformation. For grade 3, tumours show a compact stroma in which the stromal cells are universally pleomorphic and the giant cells are smaller and less numerous [42].

2.5. Immunohistochemistry. For each sample, paraffinembedded sections were cut $3 \mu \mathrm{m}$ thin, dewaxed in xylene, rehydrated, and submitted to antigen retrieval in EDTA buffer ( $\mathrm{pH}$ 9.0) or citrate ( $\mathrm{pH}$ 6.0). Each slide was blocked for endogenous peroxidase activity through incubation in $\mathrm{H}_{2} \mathrm{O}_{2} 3 \%$ for $5 \mathrm{~min}$, followed by $60 \mathrm{~min}$ incubation at ambient temperature with anti-Her2 (mouse monoclonal antibody, clone CB11, dilution 1:400, Leica Biosystems) and anti-EGFR (mouse monoclonal antibody, clone EGFR.113, dilution 1:20, Leica Biosystems). For RANKL detection, an overnight incubation at $4^{\circ} \mathrm{C}$ with a $5 \mu \mathrm{g} / \mathrm{ml}$ working solution of an anti-RANKL (mouse monoclonal antibody $\operatorname{IgG}_{2 \mathrm{~B}}$, clone: 70525, MAB626, antibody, R\&D Systems) was adopted. Post Primary (rabbit anti-mouse IgG) was then used to detect mouse antibodies. Sections were incubated with the Novolink ${ }^{\mathrm{TM}}$ Polymer that recognizes rabbit 
immunoglobulins (Novolink ${ }^{\mathrm{TM}}$ Polymer Detection System, RE7290-K) and further incubated with the substrate/chromogen, 3,3' -diaminobenzidine (DAB), prepared from DAB chromogenanaNovolink ${ }^{\mathrm{TM}}$ DAB substrate Buffer (polymer). Reactions with the peroxidase produced a visible brown precipitate at the antigen site. Sections were counterstained with hematoxylin and coverslipped. Immunohistochemical staining was evaluated according to intensity; intensities were scored as 0 , no staining; + , weak staining; ++ , moderate staining; or +++ , strong staining. Positive and negative controls were used to validate antibodies.

\subsection{Mutational Analysis}

2.6.1. DNA Samples. Fresh-frozen surgically resected samples were collected from GCTB patients. Genomic DNA was extracted from tumour tissues using phenol-chloroform standard procedures.

(1) Sample Preparation and Phenol/Chloroform Extraction of $D N A$. Frozen GCTB tissues were cut up to $3 \mu \mathrm{m}$ into small chips (2-4) using a cryostat and placed in a sterile tube. Tissues are disaggregated and treated with PK-buffer $(2 \mathrm{M}$ Tris- $\mathrm{HCl}, 0.5 \mathrm{M}$ EDTA, $1.5 \mathrm{M} \mathrm{NaCl}$ ) without Sodium Dodecyl Sulphate (SDS). The tissue was homogenized while it is submerged in PK-buffer using a syringe until complete homogenization. $20 \mu \mathrm{l}$ SDS was added then vortexed and resuspended in $50 \mu \mathrm{l}$ proteinase $\mathrm{K}(10 \mathrm{mg} / \mathrm{ml}$ stock; HiMedia). An equal volume of phenol: chloroform : isoamyl alcohol was added and mixed by inverting the tube for $20 \mathrm{~min}$ and centrifuging at $2500 \mathrm{tr} / \mathrm{min}$ (at $4^{\circ} \mathrm{C}$ ) for $10 \mathrm{~min}$. Chloroform is then used to facilitate the removal of phenol. The sample was incubated overnight at $-20^{\circ} \mathrm{C}$. DNA is subsequently purified by precipitation in ethanol. Finally, DNA is resolubilized in Tris-EDTA buffer.

2.6.2. Sequencing of the PIK3CA, PTEN, and KRAS Genes. The exons 9 and 20 of PIK3CA, exons 5 and 7 of PTEN, and exon 2 of KRAS genes were amplified by PCR, using primers and PCR condition described previously. After PCR amplification, each PCR product was purified and subsequently analysed by direct sequencing in an ABI PRISM 3100-avant automated DNA sequencer, by means of the Big Dye Terminator Cycle Sequencing reaction kit v1.1. The resulted sequences were compared to human genomic DNA sequence using blast, and the mutations were identified using ENSEMBL and Cosmic database.

\subsection{Computational Analysis}

2.7.1. The Sequence Alignment. A BLAST homology search was performed using the program BLAST2SEQ available at the NCBI (National Center for Biotechnology Information) website (https://www.ncbi.nlm.nih.gov/blast/bl2seq) to compare individual nucleotide sequences with the human gene wild-type sequence.

The sequence alignment was performed using the ClustalW program (http://bioinfo.hku.hk/services/analyseq/cgibin/clustalw_in.pl).
2.7.2. Protein Structure Prediction. Structures are visualized, and figures were generated using the Biovia Studio visualizer (Accelrys Software Inc., Discovery Studio Modeling Environment, Release 2017 R2, San Diego: Accelrys Software Inc., 2007). The structure of the complex between P110 and nSH2-iSH2 domains of regulatory subunit taken as wildtype protein has the pdb code 4L23 (https://www.rcsb.org/). The mutant E545A was generated using the Biovia Studio visualizer by replacing E545 in the wild-type structure by alanine. The same software was used to optimize A545 configuration and to check the absence of any clashes with the surrounding residues.

2.8. Statistical Analysis. The SPSS statistical package (version 20.0; SPSS, USA) was employed for statistical analysis. Pearson's chi-squared or Fisher tests were used to evaluate the association between Her2, EGFR, and RANKL biomarker overexpression and GCTB recurrence. A $p$ value of $<0.05$ was considered significant.

\section{Results}

3.1. Case Selection. A total of 32 patients, 11 men and 21 women, were recruited within our Orthopaedics and Traumatology Department. Clinical and pathological examination confirmed the GCTB diagnosis. Patient age ranged between 12 and 74 years, with a majority between 20 and 40 years $(56.2 \%)$. Tumours were mainly located in the radius $10(31 \%)$, tibia $9(28 \%)$, femur $7(22 \%)$, fibula $3(9.5 \%)$, and other rare locations 3 (9.5\%). We applied 2 grading systems in order to classify the tumours. According to Campanacci's grading system, 6 tumours were stage 1, 19 stage 2 , and 7 cases stage 3 , respectively (Figure 1(a) and Table 1). According to the Jaffe-Lichtenstein grading system, 3 tumours were classified grade 1, 15 grade 2, and 14 grade 3 (Figure 1(b) and Table 1). Time of recurrence was quite disparate and ranged from 6 to 48 months and rated in $47 \%$ of the studied cases. Moreover, curettage has a significantly higher recurrence rate than wide resection. Indeed, 21 of the 32 patients studied underwent curettage as a treatment. Also, we have shown that the type of surgery is significantly associated with recurrence; in fact, among the 21 patients treated with curettage, 13 have recurrence $(p<0.005)$, and 2 among 7 patients treated with wide resection have recurrence $(p<0.005)$. Interestingly, the majority of patients with high radiological and histological grades and 10 over 15 recurrent patients with high histological grade underwent curettage as treatment. Therefore, there was no significant correlation between age, gender, and recurrence. However, according to both systems of grading and surgery methods, higher grades were significantly associated with recurrence (Table 1).

\subsection{Expression Level of HER2, EGFR, and RANKL and GCTB} Tumour Recurrence Correlation. Using immunohistochemistry, we analysed the expression level of HER2, EGFR, and RANKL in GCTB tumours. We found that HER2 was highly expressed, mainly in the cytoplasm of multinucleated giant cells corresponding to the nonneoplastic compartment (Figure 2(a)). Indeed, HER2 was expressed moderately $(++)$ 


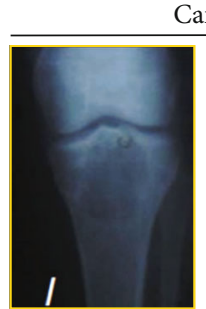

Grade 1

Campanacci grading system

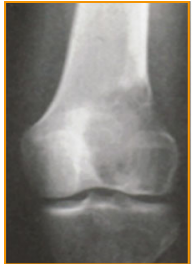

Grade 2

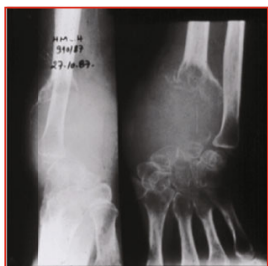

Grade 3

(a)

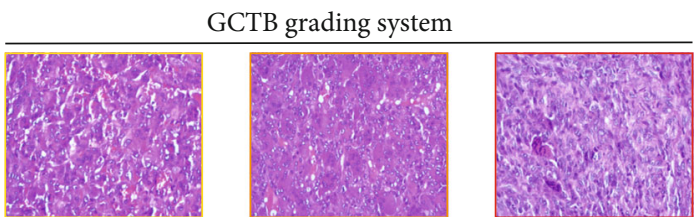

Grade 1
Grade 3
Grade 2

(b)

FIgure 1: System grading of GCTB. (a) Campanacci grading system. Tumours were classified as the following: grade 1: tumour with wellmarginated border of a thin rim of mature bone, and the cortex is intact or slightly thinned but not deformed (picture labelled in yellow). Grade 2: tumour with relatively well-defined margins but no radiopaque rim. The combined cortex and rim of reactive bone is rather thin and moderately expanded but still present (picture labelled in orange). We should note that Grade 2 lesions with a fracture are graded separately. Grade 3: tumour with fuzzy borders, suggesting a rapid and possibly permeative growth. The tumour bulges into the soft tissues, but the soft tissue mass does not follow the contour of the bone and is not limited by an apparent shell of reactive bone (picture labelled in red). (b) GCTB grades based on HE staining. Grade 1: mononuclear cells are few and not atypical with the absence of mitoses. Grade 2: more mononuclear cells with some mitotic figures. Grade 3: predominance of mononuclear cells; giant cells are few with a marked nuclear pleomorphism $(\mathrm{HE} \times 200)$.

TABLE 1: Univariate analysis of prognostic factors in GCTB.

\begin{tabular}{|c|c|c|c|c|}
\hline Categories & With recurrence & Without recurrence & Total & $p$ value \\
\hline Cases, $n(\%)$ & $15(47 \%)$ & $17(53 \%)$ & 32 & \\
\hline Sex, $n(\%)$ & & & & $p>0,05$ \\
\hline Male & $3(20 \%)$ & $8(47,1 \%)$ & $11(34,4 \%)$ & \\
\hline Female & $12(80 \%)$ & $9(52,9 \%)$ & $21(65,6 \%)$ & \\
\hline Age group, $n(\%)$ & & & & $p>0,05$ \\
\hline$<20$ ans & $1(6,7 \%)$ & $3(17,6 \%)$ & $4(12,5 \%)$ & \\
\hline $20-39$ ans & $10(66,7 \%)$ & $8(47,1 \%)$ & $18(56,2 \%)$ & \\
\hline$\geq 40$ ans & $4(26,7 \%)$ & $6(35,3 \%)$ & $10(31,2 \%)$ & \\
\hline Location, $n(\%)$ & & & & $p>0,05$ \\
\hline Femur & $2(13,3 \%)$ & $5(29,4 \%)$ & $7(21,9 \%)$ & \\
\hline Tibia & $4(26,7 \%)$ & $5(29,4 \%)$ & $9(28,1 \%)$ & \\
\hline Radius & $7(46,7 \%)$ & $3(17,6 \%)$ & $10(31,2 \%)$ & \\
\hline Fibula & $1(6,7 \%)$ & $2(11,8 \%)$ & $3(9,4 \%)$ & \\
\hline Other locations & $1(6,7 \%)$ & $2(11,8 \%)$ & $3(9,4 \%)$ & \\
\hline Campanacci's grade, $n(\%)$ & & & & $p<0,05^{*}$ \\
\hline $\mathrm{I} / \mathrm{II}$ & $9(60 \%)$ & $16(94,1 \%)$ & $25(78,1 \%)$ & \\
\hline III & $6(40 \%)$ & $1(5,9 \%)$ & $7(21,9 \%)$ & \\
\hline Jaffe-Lichtenstein grade, $n(\%)$ & & & & $p<0,05^{*}$ \\
\hline $\mathrm{I} / \mathrm{II}$ & $2(14,3 \%)$ & $15(83,3 \%)$ & $17(53,1 \%)$ & \\
\hline III & $12(85,7 \%)$ & $3(16,7 \%)$ & $15(46,9 \%)$ & \\
\hline Surgical method, $n(\%)$ & & & & $p<0,05^{*}$ \\
\hline Biopsy & $0(0,0 \%)$ & $4(23,5 \%)$ & $4(12,5 \%)$ & \\
\hline Intralesional curettage & $13(86,7 \%)$ & $8(47,1 \%)$ & $21(65,6 \%)$ & \\
\hline Wide resection & $2(13,3 \%)$ & $5(29,4 \%)$ & $7(21,9 \%)$ & \\
\hline
\end{tabular}

${ }^{*}$ Significantly different by the chi-squared test.

in 6 patients $(18.75 \%)$ and strongly $(+++)$ in 26 patients (81.25\%). GCTB recurrence was found in 4 over 6 patients with moderate expression and 14 over 26 in patients with strong expression. However, there was no significant correla- tion between HER2 expression and recurrence (Table 2). We analysed then the expression level of EGFR, a strong expression level as confirmed by the high signal we found. EGFR is localized in both types of cells, within the neoplastic and the 


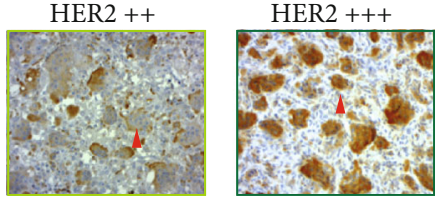

(a)

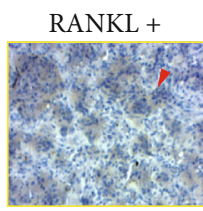

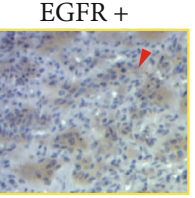

RANKL ++

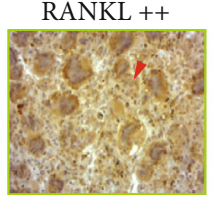

(c)
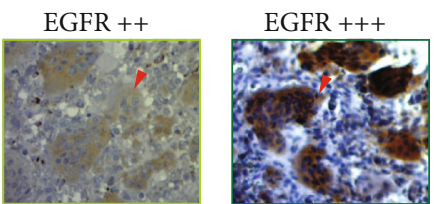

(b)

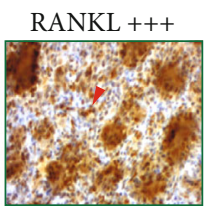

FIGURE 2: Expression level of HER2, EGFR, and RANKL. (a) Expression of HER2 in GCTB using immunohistochemistry and appropriate antibodies. Moderate level (++), picture labelled in light green, and strong level (+++), picture labelled in dark green, respectively. HER2 is localized in the cytoplasm of giant cells. The arrowheads indicate positive staining $(\times 200)$. (b) Expression of EGFR in GCTB using immunohistochemistry and appropriate antibodies. Weak expression level $(+)$, picture labelled in yellow; moderate level $(++)$, picture labelled in light green; and strong level $(+++)$, picture labelled in dark green, respectively. EGFR is localized in both types of cells, giant cells and some mononuclear cells. The arrowheads indicate positive staining $(\times 200)$. (c) Expression of RANKL in GCTB using immunohistochemistry and appropriate antibodies. Weak expression level $(+)$, picture labelled in yellow; moderate level $(++)$, picture labelled in light green; and strong level $(+++)$, picture labelled in dark green, respectively. RANKL is localized in the cytoplasm of the stromal and giant cells. The arrowheads indicate positive staining $(\times 200)$.

TABLE 2: Correlations between Her2, EGFR, RANKL, and Her2/EGFR expression with recurrence.

\begin{tabular}{lccccc}
\hline & Parameter & With recurrence & Without recurrence & Recurrence rate & $p$ value \\
\hline \multirow{2}{*}{ Expression HER2 } & ++ & 4 & 2 & $66,66 \%$ & $p>0,05$ \\
& +++ & 14 & 12 & $53,84 \%$ & \\
Expression EGFR & ++++ & 6 & 13 & $31,57 \%$ & $p<0,05^{*}$ \\
& +++ & 9 & 4 & $69,23 \%$ & \\
Expression RANKL & $+/++$ & 8 & 15 & $34,78 \%$ & $p<0,05^{*}$ \\
& +++ & 7 & 13 & $77,77 \%$ & \\
Over EGFR/Her2 & Negative & 6 & 4 & $69,57 \%$ & $p<0,05^{*}$ \\
& Positive & 9 & & & \\
\hline
\end{tabular}

${ }^{*}$ Significantly different by the chi-squared test.

nonneoplastic compartments (Figure 2(b)). Distribution over tumour samples was as follows: weak expression $(+)$ in 3 patients $(9.375 \%)$, moderate expression $(++)$ in 16 patients (50\%), and strong expression (+++) in 13 patients (40.625\%). Interestingly, we found that $70 \%$ of patients with strong staining are getting tumour recurrence, and thus, a significant positive correlation between EGFR overexpression and recurrence was confirmed. Moreover, 13 patients displayed an associated overexpression of EGFR and HER2 with a statistically significant tumour recurrence (Table 2). A contrario of HER2, RANKL was essentially observed in the neoplastic compartment of GCTB. We found that RANKL is weakly expressed in 9 patients $(28.125 \%)$, moderately in 14 (53.125\%), and strongly in 9 patients (18.75\%) (Figure 2(c)). In addition, $77 \%$ of patients with strong expression were getting tumour recurrence (Table 2).

3.3. Mutational and Bioinformatics Analysis of GCTB Tumours. Based on key oncogenes described in Introduction, we decided to perform a genetic study focusing on PIK3CA, PTEN, and KRAS genes. We sequenced these genes in all our
GCTB patients. Interestingly, we did not found any mutations in exons 5 and 7 of PTEN gene, in exon 2 of KRAS gene, and in exon 20 of PIK3CA gene. However, we found that the PIK3CA gene harbours two mutations in exon 9. First, a missense mutation, c.1634A $>C$ encoding p.E545A, substitutes the highly conserved "Glutamic acid, E" by "alanine, A" in the position 545, p.E545A within the helical domain (Figures 3(a) and 3(b)). As the missense mutation could affect the PIK3CA protein structure, we decided to perform a bioinformatics analysis on both wild-type and mutant protein. For this purpose, we used the program Biovia Studio visualizer (Accelrys Software Inc., Discovery Studio Modeling Environment, Release 2017 R2, San Diego: Accelrys Software Inc., 2007). In the wild-type protein, E545 belonging to the PIK3CA (p110) is establishing ionic interaction with $\mathrm{K} 379$ of the nSH2 domain of the regulatory subunit (p85) (Figure 3(c), i and ii). E545 is also ion pairing with the close K548 of P110. The NH group of E545 is stabilized by hydrogen bonding with the carbonyl group of L380. A replacement of E545 by alanine disrupted the ion pairing with K379 without disrupting the complex. This would lead to lower affinity 


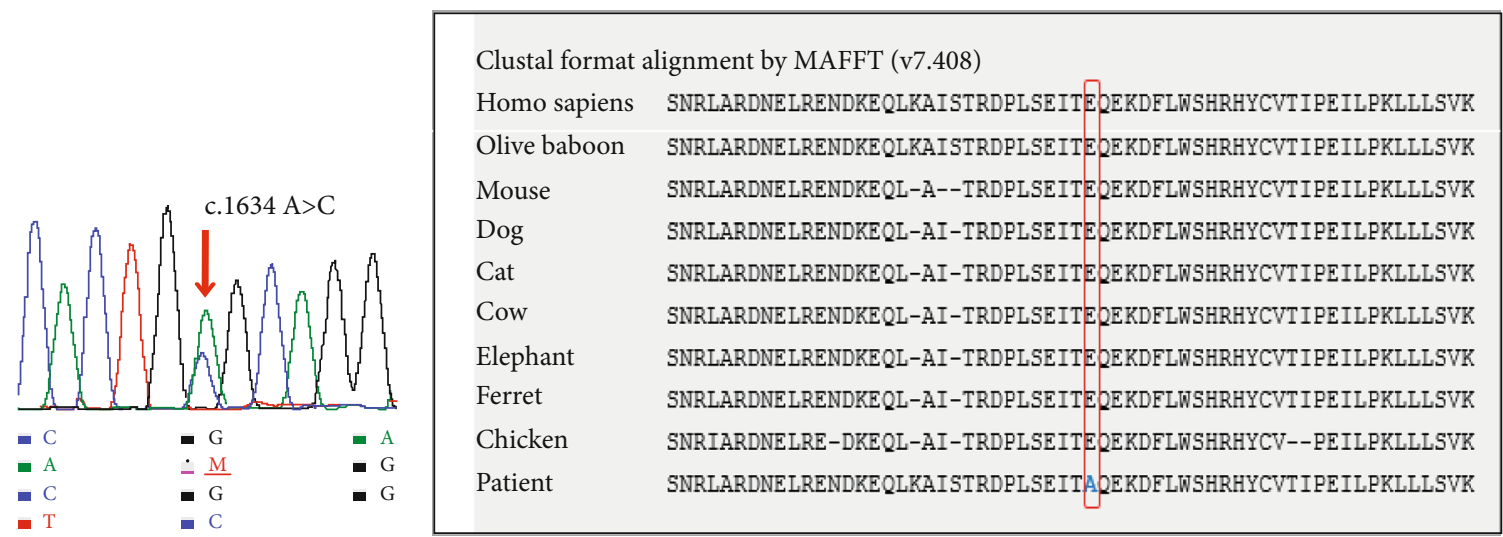

(a)

(b)

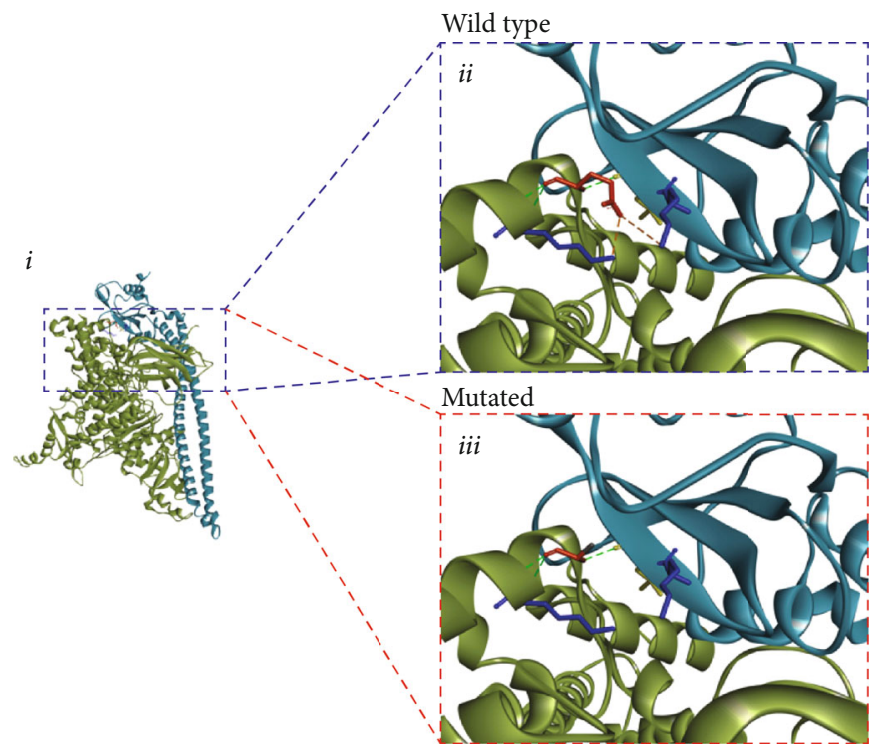

(c)

FIgURE 3: Missense mutation, c.1634A $>$ C encoding p.E545A, in exon 9 of PIK3CA gene. (a) Sequence chromatograms from affected patient with the c.1634A $>$ C mutation in PIK3CA gene. (b) Sequence alignment of the PIK3CA protein in different species performed by the ClustalW program showing the conservation of the residue Glutamic acid (E) at position 545. (c) Interaction between the catalytic subunit p110 of PI 3 kinase and the niSH2 domain of the regulatory p85 subunit (pdb code 4L23). (i) Overall cartoon structure of the complex, (ii) zoom of the amino acid E545 (red sticks) and its ionic interactions (in orange discontinued line) with K548 of P110 and K379 of p85 subunit (nSH2 domain), and (iii) E545A mutant showing A545 interacting with L380 of the regulatory subunit.

between the two proteins, but the interaction would remain due to the large patch of amino acids involved in the complex stabilization. Like the wild-type protein, E545A mutant is also stabilized by hydrogen bonding with L380. Furthermore, the nondisturbance of the interaction between the regulatory and the catalytic subunits of PI3K by this mutation is very likely due to the small side chain of alanine residue preventing clashes with surrounding residues. A contrario, Ala545 in the mutated protein is even stabilized through hydrophobic contacts with Leu380 and Ile381 belonging to the regulatory subunit (Figure 3(c), i and iii). Altogether, these observations explain a lack of effect of the mutation E545A on the PIK3CA regulation by the regulatory subunit.

The second mutation we found is a frameshift mutation, c.1658_1659delGTinsC encoding p.S553Tfs*7. It is a deletion of the dinucleotide GT plus an insertion of a $\mathrm{C}$ resulting in a serine-to-threonine substitution at codon 553, followed by frameshift in a stop codon, 7 amino acids later. These changes induced the creation of a new premature stop codon (TAA) at position 559 and cause the loss of the PI3K protein catalytic domain (Figures 4(a)-4(c)).

\section{Discussion}

In this study, we aimed to determine the risk factors for GCTB recurrence in a cohort of patients and to investigate the HER2, EGFR, and RANKL expression within the GCTB, in relationship with tumour recurrence. We aimed also to evaluate the involvement of PI3K/AKT and MAPK pathways in GCTB development.

Within our cohort, we found a feminine predominance that confirms previous reports [43]. This predominance is 


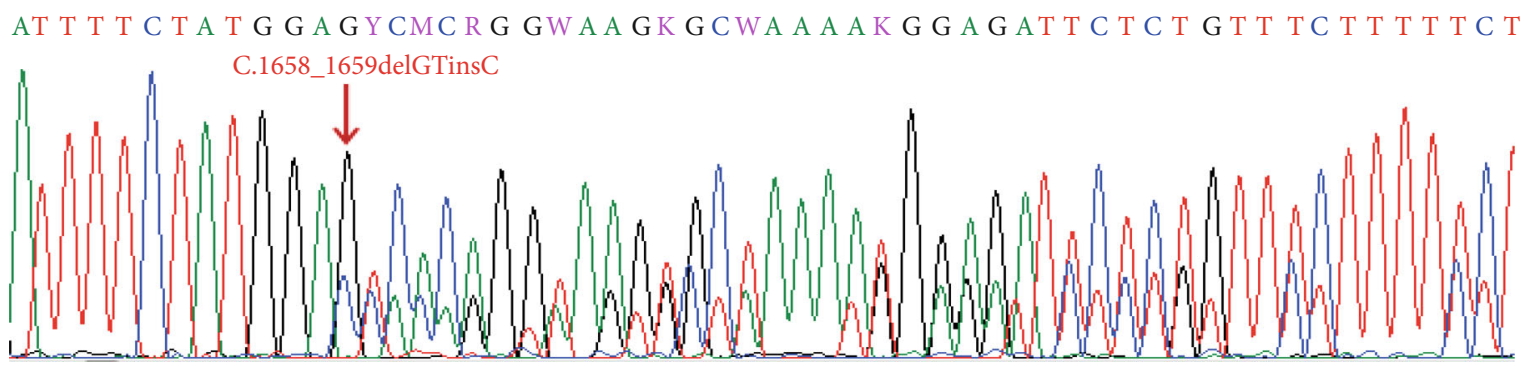

(a)

$\underline{\text { Normal protein (S553) }}$

\begin{tabular}{|c|c|c|c|c|c|c|c|}
\hline TGG & $\mathrm{AGT}$ & CAC & AGA & CAC & TAT & TGT & $\mathrm{G}^{-}$ \\
\hline W & $S$ & $\mathrm{H}$ & $\mathrm{R}$ & $\mathrm{H}$ & Y & C & \\
\hline 552 & 553 & 554 & 555 & 556 & 557 & 558 & \\
\hline \multicolumn{8}{|c|}{ Truncated protein (S553Tfs*7) } \\
\hline TGG & ACC & ACA & GAC & ACT & ATT & GTT & \\
\hline W & $\mathrm{T}$ & $\mathrm{T}$ & D & $\mathrm{T}$ & I & V & \\
\hline 553 & 554 & 555 & 556 & 557 & 558 & 559 & \\
\hline
\end{tabular}

(b)

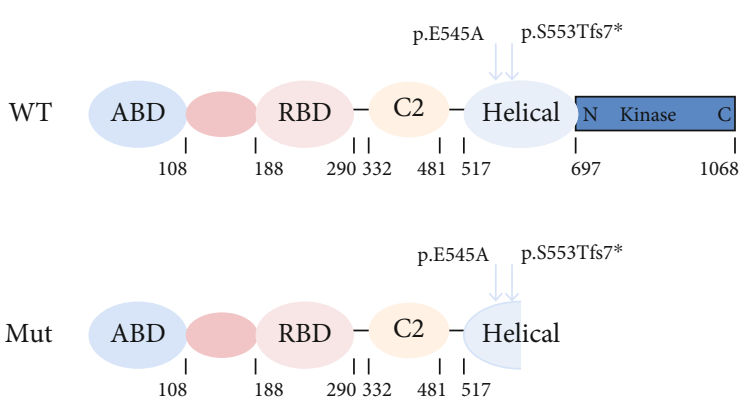

(c)

Figure 4: Frameshift mutation, c.1658-1659delGTinsC encoding p.S553Tfs $* 7$, in the exon 9 of PIK3CA gene. (a) Sequence chromatograms from affected patient with the c.1658-1659delGTinsC mutation in PIK3CA gene. (b) c.1658-1659delGTinsC substituted the serine residue (S) at position 1658 to threonine residue $(\mathrm{T})$ and changed the 7 amino acids next to the insertion leading to a premature termination codon (p.S553Tfs7X). (c) Loss of the catalytic domain of PIK3CA protein in the mutated form.

not correlated with GCTB tumour recurrence. However, a recent study suggested that recurrence should be more considered in men with GCTB than women [44]. In our study, no significant correlation was found between age and recurrence despite the study of $\mathrm{Hu}$ et al. that revealed a positive association between age and recurrence (2016) [45]. Similarly, our analysis revealed that location is not associated with recurrence while others reported that localization in the radius and proximal tibia was more frequently associated with tumour recurrence [46]. Grading systems Campanacci and Jaffe revealed a strong correlation between tumour grade and recurrence in our cohort and are in line with previous studies $[47,48]$.

We studied then the expression of HER 2 and found that over $80 \%$ of the analysed tumours overexpressed the protein. HER2 expression was exclusively cytoplasmic, and no concomitant membranous immunostaining has been detected in giant cells. Multinucleated giant cells are "reactive" components and originate from osteoclast precursors (bone marrow-derived monocytes) based on a resemblance in differentiation markers. Despite their nonneoplastic origin, multinuclear giant cells caused lacunar bone resorption, the major characteristics of this tumour [49]. In addition in our case, these cells are overexpressing the most oncogenic receptor in solid cancer (EGFR/HER2) [50]. Despite the fact that literature may qualify the absence of HER2 membrane staining, as an artefact [51], in numerous studies, overexpression of HER2 within the cytoplasm of cancer cells was a fact [52, 53]. Another study suggested that cytoplasmic immunostaining of HER2 was related to the presence of a truncated form "p95her2 [54]. Furthermore, cytoplasmic HER2 expression was described in osteosarcoma and Ewing and Synovial sarcomas $[55,56]$, and in 2005, Scotlandi et al. reported an association of HER2 expression with a worse prognosis in osteosarcoma (2005) [57].

We studied also the expression of EGFR and found that GCTB tumours exacerbate its expression in both types of compartments, in multinuclear giant cells and mononuclear neoplastic cells. Infante et al. suggested that EGFR could play as a RANK downstream regulator and/or a class of RANK coreceptors in osteoclasts (2008) [58]. It is well known that RANKL stimulation triggers the recruitment of TRAFs to RANK and the subsequent activation of the NF- $\kappa \mathrm{B}$ and MAPK cascades (ERK, JNK, and p38) with the induction of Src- and PI3K-dependent AKT activation [59]. EGFR activation could be an extra component of RANK downstream signalling, and activated EGFR could reinforce the assembly of multiprotein signalling complexes including RANK and Gab2 leading to a further activation of JNK, $\mathrm{AKT} / \mathrm{PKB}$, and NF- $\kappa \mathrm{B}$ [58]. We found also a significant correlation between overexpression of HER2/EGFR with tumour recurrence. These results confirm what has been reported in terms of correlation of HER2/EGFR to highly aggressive tumours [50, 59, 60].

We showed also that RANKL is overexpressed in our cohort, confirming what has been published previously in different tumours, including breast cancer, colorectal cancer, osteosarcoma, and GCTB $[61,62]$. We showed that RANKL is predominantly expressed in mononuclear stromal cells corresponding to the neoplastic compartment. The stromal cells regulate the formation of osteoclast-like giant cells in the neoplasm [63]. In fact, GCTB is composed of "neoplastic" 
TABLE 3: Clinical features and comparative data of patients with p.E545P and p.S553Tfs*7 mutations in several studies.

\begin{tabular}{|c|c|c|c|c|c|}
\hline & Nucleotide & Protein & Pathology & Clinical outcome & Studies \\
\hline \multirow{4}{*}{$\begin{array}{l}\text { PIK3CA } \\
\text { gene } \\
\text { Exon } 9\end{array}$} & c. $1634 \mathrm{~A}>\mathrm{C}$ & E545A & Cancer & $\begin{array}{l}\text { (i) Gain of function } \\
\text { (ii) Oncogenic transformation }\end{array}$ & $\begin{array}{l}\text { Gymnopoulus } \\
\text { et al., } 2018\end{array}$ \\
\hline & c.1658-1659GTdelinsC & S553Tfs $* 7$ & $\begin{array}{l}\text { Endometrial } \\
\text { cancer }\end{array}$ & $\begin{array}{l}\text { (i) PI3K expression: } 2+/ 80 \% \text { cells } \\
\text { (ii) pAKT: } 0\end{array}$ & $\begin{array}{l}\text { O Tredan et al., } \\
2012\end{array}$ \\
\hline & $\begin{array}{c}\text { c.1634A >C \& c.1658- } \\
\text { 1659GTdelinsC }\end{array}$ & $\begin{array}{l}\text { E545A \& } \\
\text { S553Tfs } * 7\end{array}$ & $\begin{array}{l}\text { Adenocarcinoma } \\
\text { (AC) }\end{array}$ & $\begin{array}{l}\text { (i) Stage IV } \\
\text { (ii) Overall survival: } 4 \text { months } \\
\text { (iii) He had bone metastasis at diagnosis } \\
\text { (iv) No EGFR, BRAF, and KRAS mutations }\end{array}$ & $\begin{array}{l}\text { S Ekinci et al., } \\
2015\end{array}$ \\
\hline & $\begin{array}{c}\text { c.1634A >C \& c.1658- } \\
\text { 1659GTdelinsC }\end{array}$ & $\begin{array}{c}\text { E545A \& } \\
\text { S553Tfs } * 7\end{array}$ & $\begin{array}{l}\text { Cowden } \\
\text { syndrome }\end{array}$ & $\begin{array}{l}\text { (i) Driver of predisposition to hamartoma- } \\
\text { neoplasia syndromes } \\
\text { (ii) Increase in kinase activity }\end{array}$ & Orloff et al., 2013 \\
\hline
\end{tabular}

cells with fibroblast-like spindled morphology in harmony with "reactive" macrophage-like round or osteoclast-like multinucleated giant cells $[64,65]$. Previous studies confirmed that RANK/RANKL interaction and macrophage colony-stimulating factor (M-CSF) are key players in osteoclastogenesis by recruiting mononuclear osteoclast precursor cells that differentiate into multinucleated osteoclast-like giant cells leading to bone resorption. The reciprocal and orchestrated actions between mononuclear stromal cells and multinucleated giant cells help in the understanding of the molecular pathogenesis and tumour biology of GCTB [66]. Furthermore, it was previously shown that blocking RANKL binding to RANK arrests tumour progression, improves survival, and also inhibits pulmonary metastasis [67-69].

PI3K/AKT and MAPK constitute important downstream signalling pathways of EGFR and HER2 [70]. We showed in our study that no mutations in PTEN and KRAS genes were detected in all GCTB patients. However, in PIK3CA gene, we detected two mutations in exon 9 among the helical domain: a frameshift mutation $1658_{-}$ 1659delGTinsC; p.S553Tfs $* 7$ and a missense mutation c.1634A $>C$ encoding p.E545A, and in all patients, no mutations were detected in exon 20 (encoding for the kinase domain) of PIK3CA gene.

The frameshift mutation shortens the protein and causes the loss of the PI3K protein catalytic domain. As a consequence, this mutation will interfere with the conversion of phosphatidylinositol 4,5-bisphosphate (PIP2) to phosphatidylinositol-3,4,5-triphosphate (PIP3). This PIP3 acts as a membrane anchor for the downstream proteins like PDK1 and AKT, and it seems that this mutation interferes with the PI3K/AKT antiapoptotic pathway through the inhibition of the AKT [71]. This frameshift mutation was described in a patient with endometrial cancer; it was associated to long-term nonprogressive disease as reported by Olivier Tredan (2013). IHC analysis confirmed the absence of phospho-AKT in the corresponding patient. No mutations have been proven in KRAS, PTEN, or AKT1 genes (Table 3) [72]. We can conclude that this frameshift mutation does not have any pathogenic potential.
The missense mutation does not change the protein activity, and we could speculate that E545A mutation in PIK3CA gene is not oncogenic in GCTB despite great evidences previously reported in other cancer models. Indeed, previous studies were pretending that E545A mutation is described as an oncogenic mutation in different types of cancer, such as breast and ovarian (Table 3) [73, 74]. Furthermore, Gymnopoulos et al. showed that mutations in PIK3CA, including E545A, gained function-induced oncogenic transformation (Table 3) (2007) [75].

However, some studies revealed that the coexistence of both mutations of PIK3CA, E545A, and S553T fs $* 7$ has a pathogenic potential in the Cowden syndrome, which is known to be a cancer predisposition syndrome. In fact, the heterozygosis for these two mutations in exon 9 of the PIK3CA gene led to an increase in kinase activity [76]. This pathogenic potential induced by the coexistence of both mutations was described also in non-small-cell lung cancer (NSCLC) with a locally advanced stage and with stage IV (Table 3). Therefore, the poor prognosis and the resistance to treatment in these patients might be related to other genetic alterations and factors [77]. In our patients therefore, no pathogenicity was recorded despite the coexistence of the two mutations in PIK3CA gene.

\section{Conclusions}

The present study is an attempt to identify possible criteria for predicting recurrence of Giant Cell Tumour of Bone. In addition to generally known parameters, like localization of the lesion, clear destruction of cortex with the presence of extra compartmental lesion, and histological criteria for malignancy, we have found the following independent predictors, namely, the overexpression of HER2 and EGFR in the recurrent group. The correlation between HER2/EGFR overexpression with tumour recurrence suggested that HER2/EGFR signalling plays a crucial role in aggressiveness and recurrence in GCTB. However, despite the occurrence of mutations in the protooncogenic PIK3CA gene, PI3K and MAPK pathways may not play a crucial role in the pathogenesis of GCTB. 


\section{Data Availability}

All data generated or analyzed during this study are included in this article. All data and materials are presented in Materials and Methods and Results as shown in figures and tables.

\section{Conflicts of Interest}

All authors declare that there is no conflict of interest.

\section{Acknowledgments}

This work was funded by the Tunisian Ministry of Higher Education and Scientific Research. The authors thank the patients and their families for their cooperation in the present study.

\section{References}

[1] H. Urakawa, T. Yonemoto, S. Matsumoto et al., "Clinical outcome of primary giant cell tumor of bone after curettage with or without perioperative denosumab in Japan: from a questionnaire for JCOG 1610 study," World Journal of Surgical Oncology, vol. 16, no. 1, p. 160, 2018.

[2] M. A. Ghert, M. Rizzo, J. M. Harrelson, and S. P. Scully, "Giant-cell tumor of the appendicular skeleton," Clinical Orthopaedics and Related Research, vol. 400, pp. 201-210, 2002.

[3] A. E. Horvai, M. J. Kramer, J. J. Garcia, and R. J. O'Donnell, "Distribution and prognostic significance of human telomerase reverse transcriptase (hTERT) expression in giant-cell tumor of bone," Modern Pathology, vol. 21, no. 4, pp. 423430, 2008.

[4] S. Chawla, J.-Y. Blay, P. Rutkowski et al., "Denosumab in patients with giant-cell tumour of bone: a multicentre, openlabel, phase 2 study," The Lancet Oncology, vol. 20, no. 12, pp. 1719-1729, 2019.

[5] M. Szendröi, "Giant-cell tumour of bone," The Journal of Bone and Joint Surgery. British volume, vol. 86-B, no. 1, pp. 5-12, 2004.

[6] S. E. Larsson, R. Lorentzon, and L. Boquist, "Giant-cell tumor of bone. A demographic, clinical, and histopathological study of all cases recorded in the Swedish Cancer Registry for the years 1958 through 1968," The Journal of Bone \& Joint Surgery, vol. 57, no. 2, pp. 167-173, 1975.

[7] L. del Carmen Baena-Ocampo, L. M. Rosales Olivares, N. M. Arriaga, A. Izaguirre, and C. Pineda, "Pigmented villonodular synovitis of thoracic facet joint presenting as rapidly progressive paraplegia," JCR: Journal of Clinical Rheumatology, vol. 15, no. 8, pp. 393-395, 2009.

[8] J. Rockberg, B. A. Bach, J. Amelio et al., "Incidence Trends in the Diagnosis of Cell Tumor of Bone in Sweden Since 1958," The Journal of Bone and Joint Surgery, vol. 97, no. 21, pp. 1756-1766, 2015.

[9] W. Guo, W. Xu, A. G. Huvos, J. H. Healey, and C. Feng, “Comparative frequency of bone sarcomas among different racial groups," Chinese Medical Journal, vol. 112, no. 12, pp. 1101$1104,1999$.

[10] H. W. Sung, D. P. Kuo, W. P. Shu, Y. B. Chai, C. C. Liu, and S. M. Li, "Giant-cell tumor of bone: analysis of two hundred and eight cases in Chinese patients," The Journal of Bone and Joint Surgery. American Volume, vol. 64, no. 5, pp. 755-761, 1982.

[11] K. M. Skubitz, "Giant cell tumor of bone: current treatment options," Current Treatment Options in Oncology, vol. 15, no. 3, pp. 507-518, 2014.

[12] K. Namba, M. Nishio, K. Mori et al., "Involvement of ADAM9 in multinucleated giant cell formation of blood monocytes," Cellular Immunology, vol. 213, no. 2, pp. 104-113, 2001.

[13] G. J. Atkins, D. R. Haynes, S. E. Graves et al., "Expression of osteoclast differentiation signals by stromal elements of giant cell tumors," Bone and Mineral, vol. 15, no. 4, pp. 640-649, 2000.

[14] D. Robinson, M. Segal, and Z. Nevo, "Giant cell tumor of bone," Pathobiology, vol. 70, no. 6, pp. 333-342, 2003.

[15] D. D. Cheng, T. Hu, H. Z. Zhang, J. Huang, and Q. C. Yang, "Factors affecting the recurrence of giant cell tumor of bone after surgery: a clinicopathological study of 80 cases from a single center," Cell PhysiolBiochem, vol. 36, no. 5, pp. 19611970, 2015.

[16] S. Matsubayashi, M. Nakashima, K. Kumagai et al., "Immunohistochemical analyses of $\beta$-catenin and cyclin D1 expression in giant cell tumor of bone (GCTB): a possible role of Wnt pathway in GCTB tumorigenesis," Pathology, Research and Practice, vol. 205, no. 9, pp. 626-633, 2009.

[17] J. Zhang, J. Dong, Z. Yang et al., "Expression of ezrin, CD44, and VEGF in giant cell tumor of bone and its significance," World Journal of Surgical Oncology, vol. 13, no. 1, p. 168, 2015.

[18] F. Fazioli, G. Colella, R. Miceli et al., "Post-surgery fluids promote transition of cancer stem cell-to-endothelial and AKT/mTOR activity, contributing to relapse of giant cell tumors of bone," Oncotarget, vol. 8, no. 49, pp. 85040-85053, 2017.

[19] M. J. Jung, C. G. Woo, S. Lee et al., "Gene copy number variation and protein overexpression of EGFR and HER2 in distal extrahepatic cholangiocarcinoma," Pathology, vol. 49, no. 6, pp. 582-588, 2017.

[20] A. Kılıçarslan, H. T. Dogan, N. Süngü, M. Dogan, A. Yalcin, and D. S. Dede, "Association between Her2/neu status in colorectal carcinoma and clinicopathological features: a retrospective study using whole - tissue sections," Polish Journal of Pathology, vol. 69, no. 2, pp. 143-149, 2018.

[21] C. L. Gaston, R. J. Grimer, M. Parry et al., "Current status and unanswered questions on the use of Denosumab in giant cell tumor of bone," Clinical Sarcoma Research, vol. 6, no. 1, p. 15, 2016.

[22] H. Liu, Y. Dong, Y. Gao et al., "Hesperetin suppresses RANKL-induced osteoclastogenesis and ameliorates lipopolysaccharide-induced bone loss," Journal of Cellular Physiology, vol. 234, no. 7, pp. 11009-11022, 2018.

[23] D. Lin, L. Li, Y. Sun et al., "Interleukin-17 regulates the expressions of RANKL and OPG in human periodontal ligament cells via TRAF6/TBK1-JNK/NF- $\kappa$ B pathways," Journal of Immunology, vol. 144, no. 3, pp. 472-485, 2015.

[24] K. Okamoto, T. Nakashima, M. Shinohara et al., "Osteoimmunology: the conceptual framework unifying the immune and skeletal systems," Physiological Reviews, vol. 97, no. 4, pp. 1295-1349, 2017.

[25] M. G. Ruocco, S. Maeda, J. M. Park et al., "I $\kappa$ B kinase (IKK) $\beta$, but not IKK $\alpha$, is a critical mediator of osteoclast survival and 
is required for inflammation-induced bone loss," The Journal of Experimental Medicine, vol. 201, no. 10, pp. 1677-1687, 2005.

[26] A. Mukherjee, E. M. Wilson, and P. Rotwein, "Selective signaling by Akt 2 promotes bone morphogenetic protein 2mediated osteoblast differentiation," Journal of Molecular Cell Biology, vol. 30, no. 4, pp. 1018-1027, 2010.

[27] R. W. Naumann, "The role of the phosphatidylinositol 3kinase (PI3K) pathway in the development and treatment of uterine cancer," Journal of Gynecologic Oncology, vol. 123, no. 2, pp. 411-420, 2011.

[28] L. C. Cantley and B. G. Neel, "New insights into tumor suppression: PTEN suppresses tumor formation by restraining the phosphoinositide 3-kinase/AKT pathway," Proceedings of the National Academy of Sciences, vol. 96, no. 8, pp. 42404245, 1999.

[29] M. Cully, H. You, A. J. Levine, and T. W. Mak, "Beyond PTEN mutations: the PI3K pathway as an integrator of multiple inputs during tumorigenesis," Nature Reviews Cancer, vol. 6, no. 3, pp. 184-192, 2006.

[30] D. Bonneau and M. Longy, "Mutations of the human PTEN gene," Human Mutation, vol. 16, no. 2, pp. 109-122, 2000.

[31] R. Wadhwa, S. Song, J.-S. Lee, Y. Yao, Q. Wei, and J. A. Ajani, "Gastric cancer-molecular and clinical dimensions," Nature Reviews Clinical Oncology, vol. 10, no. 11, pp. 643-655, 2013.

[32] A. E. Karnoub and R. A. Weinberg, "Ras oncogenes: split personalities," Nature Reviews Molecular Cell Biology, vol. 9, no. 7, pp. 517-531, 2008.

[33] K. Lai, M. C. Killingsworth, and C. S. Lee, "Gene of the month: PIK3CA," Journal of Clinical Pathology, vol. 68, no. 4, pp. 253257, 2015.

[34] F. Perrone, A. Lampis, M. Orsenigo et al., "PI3KCA/PTEN deregulation contributes to impaired responses to cetuximab in metastatic colorectal cancer patients," Annals of Oncology, vol. 20, no. 1, pp. 84-90, 2009.

[35] S. C. Drury, S. Detre, A. Leary et al., "Changes in breast cancer biomarkers in the IGF1R/PI3K pathway in recurrent breast cancer after tamoxifen treatment," Endocrine-Related Cancer, vol. 18, no. 5, pp. 565-577, 2011.

[36] N. Karachaliou, C. Mayo, C. Costa et al., "KRAS mutations in lung cancer," Clinical Lung Cancer, vol. 14, no. 3, pp. 205-214, 2013.

[37] M. K. H. Maus, P. P. Grimminger, P. C. Mack et al., "KRAS mutations in non-small-cell lung cancer and colorectal cancer: implications for EGFR-targeted therapies," Lung Cancer, vol. 83, no. 2, pp. 163-167, 2014.

[38] N. Normanno, C. Pinto, F. Castiglione et al., "KRAS mutations testing in colorectal carcinoma patients in Italy: from guidelines to external quality assessment," PLoS One, vol. 6, no. 12, p. e29146, 2011.

[39] K. L. Bryant, J. D. Mancias, A. C. Kimmelman, and C. J. der, "KRAS: feeding pancreatic cancer proliferation," Trends in Biochemical Sciences, vol. 39, no. 2, pp. 91-100, 2014.

[40] H. Ayatollahi, A. Tavassoli, A. H. Jafarian et al., "KRAS Codon 12 and 13 Mutations in Gastriccancer in the Northeast Iran," Iranian Journal of Pathology, vol. 13, no. 2, pp. 167-172, 2018.

[41] A. Sobti, P. Agrawal, S. Agarwala, and M. Agarwal, "Giant cell tumor of bone - an overview," Archives of Bone and Joint Surgery, vol. 4, no. 1, pp. 2-9, 2016.

[42] H. Jaffe, L. Lichtenstein, and R. Portis, "Giant cell tumor of bone: its pathological appearance, grading, supposed variants and treatment," Archives of Pathology, vol. 30, pp. 993-1031, 1940.

[43] J. M. Amelio, J. Rockberg, R. K. Hernandez et al., "Populationbased study of giant cell tumor of bone in Sweden (19832011)," Cancer Epidemiology, vol. 42, pp. 82-89, 2016.

[44] Y. Hu, L. Zhao, H. Zhang et al., "Sex Differences in the Recurrence Rate and Risk Factors for Primary Giant Cell Tumors Around the Knee in China," Scientific Reports, vol. 6, no. 1, p. 28173, 2016.

[45] P. Hu, L. Zhao, H. Zhang et al., "Recurrence Rates and Risk Factors for Primary Giant Cell Tumors around the Knee: A Multicentre Retrospective Study in China," Scientific Reports, vol. 6, no. 1, p. 36332, 2016.

[46] M. A. Siddiqui, C. Seng, and M. H. Tan, "Risk factors for recurrence of giant cell Tumours of bone," Journal of Orthopaedic Surgery, vol. 22, no. 1, pp. 108-110, 2014.

[47] L. Gong, W. Liu, X. Sun et al., "Histological and clinical characteristics of malignant giant cell tumor of bone," Virchows Archiv, vol. 460, no. 3, pp. 327-334, 2012.

[48] Y. Liu, A. Moro, K. Wang et al., "Residual bone fragments in tibiofibular joint and postoperative local recurrence: an analysis of 21 cases of proximal fibular giant cell tumour," World Journal of Surgical Oncology, vol. 16, no. 1, p. 228, 2018.

[49] L. van der Heijden, P. D. S. Dijkstra, J.-Y. Blay, and H. Gelderblom, "Giant cell tumour of bone in the denosumab era," European Journal of Cancer, vol. 77, pp. 75-83, 2017.

[50] K. Imai and A. Takaoka, "Comparing antibody and smallmolecule therapies for cancer," Nature Reviews Cancer, vol. 6, no. 9, pp. 714-727, 2006.

[51] I. Busmanis, F. Feleppa, A. Jones et al., "Analysis of cerbB2 expression using a panel of 6 commercially available antibodies," Pathology, vol. 26, no. 3, pp. 261-267, 1994.

[52] E. J. Blok, P. J. K. Kuppen, J. E. M. van Leeuwen, and C. F. M. Sier, "Cytoplasmic Overexpression of HER2: a Key Factor in Colorectal Cancer," Clinical Medicine Insights: Oncology, vol. 7, article CMO.S10811, 2013.

[53] S.-i. Horiguchi, T. Hishima, Y. Hayashi et al., "HER-2/neu cytoplasmic staining is correlated with neuroendocrine differentiation in breast carcinoma," Journal of Medical and Dental Sciences, vol. 57, no. 2, pp. 155-163, 2010.

[54] L. Albarello, L. Pecciarini, and C. Doglioni, "HER2 testing in gastric cancer," Advances in Anatomic Pathology, vol. 18, no. 1, pp. 53-59, 2011.

[55] P. G. Nuciforo, C. Pellegrini, R. Fasani et al., "Molecular and immunohistochemical analysis of HER2/neu oncogene in Synovial sarcoma," Human Pathology, vol. 34, no. 7, pp. 639-645, 2003.

[56] R. Gorlick, A. G. Huvos, G. Heller et al., "Expression of HER2/erbB-2 correlates with survival in osteosarcoma," Journal of Clinical Oncology, vol. 17, no. 9, pp. 2781-2788, 1999.

[57] K. Scotlandi, M. C. Manara, C. M. Hattinger et al., "Prognostic and therapeutic relevance of HER2 expression in osteosarcoma and Ewing's sarcoma," European Journal of Cancer, vol. 41, no. 9, pp. 1349-1361, 2005.

[58] M. Infante, A. Fabi, F. Cognetti, S. Gorini, M. Caprio, and A. Fabbri, "RANKL/RANK/OPG system beyond bone remodeling: involvement in breast cancer and clinical perspectives," Journal of Experimental \& Clinical Cancer Research, vol. 38, no. 1, p. 12, 2019.

[59] Y. Yarden, "Biology of HER2 and its importance in breast cancer," Oncology, vol. 61, no. 2, pp. 1-13, 2001. 
[60] C. D. Fichter, S. Timme, J. A. Braun et al., "EGFR, HER2 and HER3 dimerization patterns guide targeted inhibition in two histotypes of esophageal cancer," International Journal of Cancer, vol. 135, no. 7, pp. 1517-1530, 2014.

[61] D. Santini, G. Schiavon, B. Vincenzi et al., "Receptor activator of NF-kB (RANK) expression in primary tumors associates with bone metastasis occurrence in breast cancer patients," PLoS One, vol. 6, no. 4, article e19234, 2011.

[62] N. E. Kushlinskii, E. S. Gershtein, Y. N. Solov'ev et al., "Receptor Activator of Nuclear Transcription Factor NF- $\kappa$ B (RANK), Its Ligand RANKL, and Natural Inhibitor of RANKLosteoprotegerin (OPG) in the Blood Serum of Patients with Primary Bone Tumors," Bulletin of Experimental Biology and Medicine, vol. 163, no. 4, pp. 478-481, 2017.

[63] L. Huang, X. Y. Teng, Y. Y. Cheng, K. M. Lee, and S. M. Kumta, "Expression of preosteoblast markers and Cbfa-1 and Osterix gene transcripts in stromal tumour cells of giant cell tumour of bone," Bone, vol. 34, no. 3, pp. 393-401, 2004.

[64] D. G. Branstetter, S. D. Nelson, J. C. Manivel et al., "Denosumab induces tumor reduction and bone formation in patients with giant-cell tumor of bone," Clinical Cancer Research, vol. 18, no. 16, pp. 4415-4424, 2012.

[65] S. Chawla, R. Henshaw, L. Seeger et al., "Safety and efficacy of denosumab for adults and skeletally mature adolescents with giant cell tumour of bone: interim analysis of an open-label, parallel-group, phase 2 study," The Lancet Oncology, vol. 14, no. 9, pp. 901-908, 2013.

[66] B. J. Noh and Y. K. Park, "Giant cell tumor of bone: updated molecular pathogenesis and tumor biology," Human Pathology, vol. 81, pp. 1-8, 2018.

[67] Y. Chen, M. A. Di Grappa, S. D. Molyneux et al., "RANKL blockade prevents and treats aggressive osteosarcomas," Journal of Cancer, vol. 7, no. 317, article 317ra197, 2015.

[68] Y. Park, "The nuclear factor-kappa B pathway and response to treatment in breast cancer," Pharmacogenomics, vol. 18, no. 18, pp. 1697-1709, 2017.

[69] I. Pettersen, W. Bakkelund, B. Smedsrød, and B. Sveinbjørnsson, "Osteoprotegerin is expressed in colon carcinoma cells," Anticancer Research, vol. 25, no. 6B, pp. 38093816, 2005.

[70] R. Mishra, A. B. Hanker, and J. T. Garrett, "Genomic alterations of ERBB receptors in cancer: clinical implications," Oncotarget, vol. 8, no. 69, pp. 114371-114392, 2017.

[71] A. Sathe, G. Chalaud, I. Oppolzer et al., "Parallel PI3K, AKT and mTOR inhibition is required to control feedback loops that limit tumor therapy," PLoS One, vol. 13, no. 1, p. e0190854, 2018.

[72] O. Trédan, I. Treilleux, Q. Wang et al., "Predicting everolimus treatment efficacy in patients with advanced endometrial carcinoma: a GINECO group study," Targeted Oncology, vol. 8, no. 4, pp. 243-251, 2013.

[73] S. El Shamieh, F. Saleh, S. Moussa, J. Kattan, and F. Farhat, "RICTORgene amplification is correlated with metastasis and therapeutic resistance in triple-negative breast cancer," Pharmacogenomics, vol. 19, no. 9, pp. 757-760, 2018.

[74] G. Jiang, Z. Huang, S. Zhang, and L. Wang, "PIK3CA gene mutations and amplifications in Chinese patients with ovarian clear cell carcinoma," Cancer Investigation, vol. 31, no. 10, pp. 639-644, 2013.
[75] M. Gymnopoulos, M. Elsiger, and P. Vogt, "Rare cancer -specific mutations in PIK3CA show gain of function," PNAS, vol. 104, no. 13, pp. 5569-5574, 2007.

[76] M. S. Orloff, X. He, C. Peterson et al., "Germline PIK3CA and AKT1 mutations in Cowden and Cowden-like syndromes," American Journal of Human Genetics, vol. 92, no. 1, pp. 7680, 2013.

[77] S. Ekinci, H. Ilgin-Ruhi, M. Dogan et al., "Molecular spectrum of PIK3CA gene mutations in patients with nonsmall-cell lung cancer in Turkey," Genetic Testing and Molecular Biomarkers, vol. 19, no. 7, pp. 353-358, 2015. 